Check for updates

Cite this: Soft Matter, 2018, 14,3040

Received 5th January 2018, Accepted 27th March 2018 DOI: $10.1039 / \mathrm{c} 8 \mathrm{sm} 00047 f$

rsc.li/soft-matter-journal

\section{Nonlocal rheology of dense granular flow in annular shear experiments}

\author{
Zhu Tang, (D) *a Theodore A. Brzinski, (D) ${ }^{b}$ Michael Shearer (D) $^{c}$ and \\ Karen E. Daniels (D) ${ }^{a}$
}

\begin{abstract}
The flow of dense granular materials at low inertial numbers cannot be fully characterized by local rheological models; several nonlocal rheologies have recently been developed to address these shortcomings. To test the efficacy of these models across different packing fractions and shear rates, we perform experiments in a quasi-2D annular shear cell with a fixed outer wall and a rotating inner wall, using photoelastic particles. The apparatus is designed to measure both the stress ratio $\mu$ (the ratio of shear to normal stress) and the inertial number / through the use of a torque sensor, laser-cut leaf springs, and particle-tracking. We obtain $\mu(/)$ curves for several different packing fractions and rotation rates, and successfully find that a single set of model parameters is able to capture the full range of data collected once we account for frictional drag with the bottom plate. Our measurements confirm the prediction that there is a growing lengthscale at a finite value $\mu_{\mathrm{s}}$, associated with a frictional yield criterion. Finally, we newly identify the physical mechanism behind this transition at $\mu_{\mathrm{s}}$ by observing that it corresponds to a drop in the susceptibility to force chain fluctuations.
\end{abstract}

\section{Introduction}

Currently, there is no first-principles, general continuum theory of dense dry granular flow that predicts its rheological response as a function of particle size, shape, and friction. A universal form for constitutive laws describing such rheology continues to be a challenging issue, despite promising recent developments. ${ }^{1-6}$ In an empirical approach, constitutive relations are postulated based on considerations of the connection between applied stresses and the properties of flow. In the experiments described here, we test several nonlocal models under various shear and packing conditions, providing insight into not only the relative success of the models, but also a physical interpretation of the underlying mechanisms.

In a dense granular flow, it is common to characterize the flow rapidity via the dimensionless inertial number ${ }^{1}$

$$
I \equiv \frac{\dot{\gamma} d}{\sqrt{P / \rho}} .
$$

This can be interpreted ${ }^{1}$ as the ratio between a microscopic timescale $T=d / \sqrt{P / \rho}$ (the time for a particle of density $\rho$ to fall into a hole of grain size $d$, under pressure $P$ ) and a macroscopic timescale $1 / \dot{\gamma}$ (the mean deformation time due to shear rate $\dot{\gamma}$ ).

\footnotetext{
${ }^{a}$ Department of Physics, North Carolina State University, Raleigh, NC, USA.

E-mail: ztang2@ncsu.edu

${ }^{b}$ Department of Physics and Astronomy, Haverford College, Haverford, PA, USA

${ }^{c}$ Department of Mathematics, North Carolina State University, Raleigh, NC, USA
}

While the modeling of rapid flows $(I \gg 1)$ is in an advanced state, ${ }^{7,8}$ intermediate flows $(I \sim 1)$ must account for both shear and compression effects, ${ }^{9}$ and slow flows $(I \ll 1)$ remain particularly challenging to model.

For slow to intermediate flows, the dimensionless stress ratio $\mu$ is defined as the ratio between the local shear stress $\tau$ and the local pressure $P$ :

$$
\mu \equiv \frac{\tau}{P}
$$

Measurements of $\mu(I)$ were first modeled by purely local considerations: any point with $\mu \geq \mu_{\mathrm{s}}$ (the yield criterion) would be flowing, and those with $\mu<\mu_{\mathrm{s}}$ would be blocked. While these local rheologies have been broadly successful at describing fully-developed flows, ${ }^{1}$ they fail to quantitatively capture the transition from inertial to quasistatic $(I \ll 1$ but still creeping) flow, ${ }^{10}$ explain the dependence of shear band width on geometry and grain size, ${ }^{11,12}$ or describe how shear/ vibration in one region of a granular material can fluidize distant regions. ${ }^{13,14}$

Due to these limitations of local rheology, several nonlocal rheology models have recently been developed. The nonlocal rheology model proposed by Kamrin and Koval ${ }^{2,15}$ extends a local Bagnold-type granular flow law to include a Laplacian term governing the diffusion of fluidity via cooperative effects. The nonlocal rheology model of Bouzid et al. ${ }^{3,4}$ performs a gradient expansion of a general constitutive relation. In both models, a Laplacian term models the diffusion of nonlocal 
effects within the granular material, but with a different physical interpretation; we will refer to these models as cooperative and gradient, respectively. Both of these models have been welltested in simulations, ${ }^{2-4,6,16}$ but experimental verifications ${ }^{15}$ have been confined to testing the kinematics (speed profiles, including the width of the shear band) rather than directly testing the relationship between applied force and the resulting flow.

In this paper, we report the results of a successful, quantitative comparison between experimental data and each of these models. We have developed a new 2D annular shear apparatus (see Fig. 1) which is instrumented to measure the shear $(\tau)$ and normal $(P)$ boundary forces. By using photoelastic particles ${ }^{17}$ as the granular material we can either perform particle tracking (without polariscope) or measure spatiotemporal fluctuations in forces (with polariscope). These methods allow us to measure $\mu(I)$ throughout the material, and provide insight into the underlying mechanisms driving nonlocal rheology.

\subsection{Cooperative model}

The cooperative model ${ }^{2,15}$ is based on extending a local Bagnold-type granular flow law to include nonlocal effects. As with Bagnold scaling, there is assumed to be a linear relationship between shear stress and shear rate. This leads to the definition of fluidity

$$
g \equiv \frac{\dot{\gamma}}{\mu}
$$

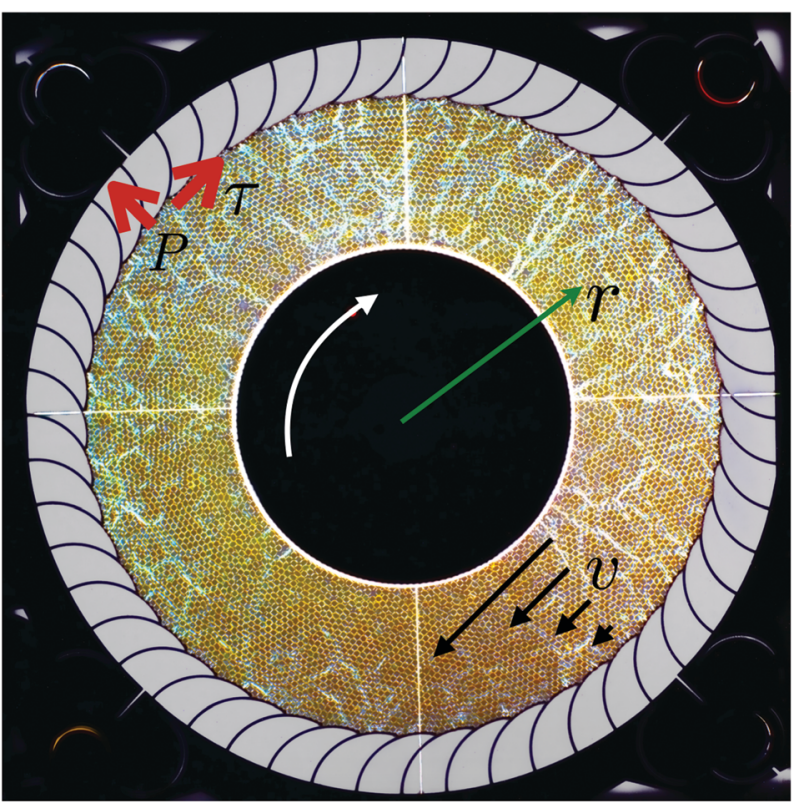

Fig. 1 Top view of annular Couette experiment with $\approx 5000$ flat photoelastic particles. Image is a composite of an image of the leaf springs and an image of the force chains, inverted for clarity (light particles are those experiencing more force). The inner wheel $\left(R_{\mathrm{i}}=15.0 \mathrm{~cm}=26.8 \mathrm{~d}\right)$ rotates at fixed speed, and the stationary outer boundary $\left(R_{\circ}=28.0 \mathrm{~cm}=50.0 \mathrm{~d}\right)$ is composed of 52 laser-cut leaf springs. The tips of the leaf springs are calibrated to measure shear $(\tau)$ and normal $(P)$ stress. The speed profile $v(r)$ is measured via particle-tracking where $\mu$ is the dimensionless stress ratio defined in eqn (2), and $g$ has units $\mathrm{s}^{-1}$. Motivated by results from numerical simulations, ${ }^{18}$ Kamrin and Koval further assumed a linear relationship between local $I$ and $\mu$, but only where $\mu$ is larger than a yield ratio $\mu_{\mathrm{s}}$. This relationship, using the Heaviside function $H(\cdot)$, is given by

$$
I(\mu)=\frac{\left(\mu-\mu_{\mathrm{s}}\right) H\left(\mu-\mu_{\mathrm{s}}\right)}{b} .
$$

The parameter $b$ controls the steepness of the rise of $I(\mu)$; in prior studies, ${ }^{2,6,16} b$ has been observed to be in the range of $1.0 \pm 0.1$. In locations where $\mu<\mu_{\mathrm{s}}$, this local rheology would predict no flow $(I=0)$. From this local flow rule and eqn (3), they define a corresponding local granular fluidity

$$
g_{\text {loc }}(\mu, P)=\frac{\left(\mu-\mu_{\mathrm{s}}\right) H\left(\mu-\mu_{\mathrm{s}}\right)}{b \mu T} .
$$

The nonlocal portion of the theory develops from assuming that the granular fluidity $g$ in eqn (3) has two contributions: the local $g_{\text {loc }}$ (eqn (5)) and a nonlocal contribution arising cooperatively from the surroundings. This is modeled as a diffusive process taking place over a cooperativity lengthscale $\xi$ :

$$
\nabla^{2} g=\frac{1}{\xi^{2}}\left(g-g_{\text {loc }}\right)
$$

For example, particle slips or vibrations or stress redistribution in one part of the system can propagate through to other parts of the system, with the system most sensitive in the vicinity of $\mu_{\mathrm{s}}$. This length scale is proposed to scale with the particle diameter $d$ via the functional form ${ }^{16}$

$$
\frac{\xi}{d}=A \sqrt{\frac{1}{\left|\mu-\mu_{\mathrm{s}}\right|}} .
$$

Note that this equation is symmetric around $\mu=\mu_{\mathrm{s}}$; this will not be true of the gradient model. The parameter $A$ controls the strength of this cooperative effect, and has been seen in previous work $^{6,16,19}$ to be in the range of $0.8 \pm 0.3$. Because $\xi$ controls the proposed mechanism of nonlocality - how one part of the system influences another - eqn (7) is a key relationship to test directly.

Prior work ${ }^{2}$ on the cooperative model originally allowed for eqn (7) to have a free exponent (found to be near 0.6) instead of the square root. Later papers ${ }^{15,16}$ chose a square root for simplicity, and we have followed that form here. More recently, the cooperative model has been extended to account for timedependent flow. ${ }^{6,16}$ In this paper, we examine steady flows and therefore take the time-independent cooperative model as our comparison.

\subsection{Gradient model}

The gradient model $^{3,4}$ takes a different definition of the fluidity

$$
f=\frac{\dot{\gamma}}{Y}
$$

where $Y \equiv \mu(I) / \mu_{\mathrm{s}}=\tau / \mu_{\mathrm{s}} P$ is the local stress ratio, relative to the same yield stress ratio $\mu_{\mathrm{s}}$ used in the cooperative model. 
This fluidity $f$ also has units of $\mathrm{s}^{-1}$, and is related to the fluidity used in the cooperative model by $f=\mu_{\mathrm{s}} g$.

The model further assumes that this expression for $Y$ is just the first (local) term in an expansion of the true (unknown) constitutive relation. The full gradient-expansion, taken in higher orders of $I$, would be

$$
Y \simeq \frac{\mu(I)}{\mu_{\mathrm{s}}}\left(1-\nu_{\ell} \kappa+\mathcal{O}\left(\kappa^{2}\right)+\cdots\right)
$$

expressed in terms of $\kappa=d^{2} \nabla^{2} I / I$. In this model, we keep only the lowest order (linear) term containing $\kappa$. This gives

$$
Y=\frac{\mu(I)}{\mu_{\mathrm{s}}}\left(1-\nu_{\ell} \frac{d^{2}\left(\nabla^{2} I\right)}{I}\right)
$$

where the phenomenological constant $\nu_{\ell}$ is a parameter controlling the magnitude of the higher-order contribution. Previous comparisons with simulations ${ }^{3}$ have observed $\nu_{\ell}=8$.

Using eqn (8) and the local definition of $Y$, eqn (10) can be written as a function of the fluidity $f$ :

$$
\dot{\gamma}=\frac{I_{\mathrm{loc}}(f)}{T}-\ell^{2} \nabla^{2} f
$$

where $T$ is again the microscopic timescale (determined from measurements of $P$ ). Physically, this corresponds to writing the shear rate $\dot{\gamma}$ as the sum of a local term and a nonlocal term. The second (Laplacian) term in eqn (11) is the nonlocal contribution, and the parameter $\ell$ represents the spatial extent of the contributions the local fluidity makes to its surroundings. Prior work has observed that $\ell$ is on the order of a few grain diameters. $^{4}$

For a homogeneously flowing steady state $\left(\mu>\mu_{\mathrm{s}}\right)$, the local inertial number $I_{\text {loc }}$ would have the same physical meaning as $I$, with a constitutive relation

$$
Y=1+a I_{\mathrm{loc}}^{n}
$$

Here, the frictional case $(n=1)$ plays the same role as eqn (4) with $\mu>\mu_{\mathrm{s}}$ in the cooperative model. We can re-write eqn (12) in terms of the fluidity $f$ by taking $Y=\dot{\gamma}_{\text {loc }} / f=I_{\text {loc }} / T f$. For $n=1$, the function $I_{\text {loc }}$ takes the form

$$
I_{\mathrm{loc}}(f)=\frac{T f}{1-a T f}
$$

where the fitting parameter $a$ controls how steeply $I_{\text {loc }}$ rises as a function of $f$. Previous comparisons with simulations ${ }^{3}$ have observed $a=4.3$.

In eqn (10), a divergent length scale $L$ arises in the vicinity of $Y=1$, due to whether or not local effects are present in $I(Y>1$ corresponds to $\mu>\mu_{\mathrm{s}}$ and vice versa.) This can be illustrated as follows. For $Y>1$, linearizing eqn (10) around a combination of local and nonlocal effects $(I+\delta I)$ gives a differential equation of the form:

$$
L^{2} \nabla^{2} I-\delta I=0
$$

The solutions of this equation are exponentials with a lengthscale given by $L=d \sqrt{\frac{Y \nu_{\ell}}{Y-1}}$. However, for $Y<1$, there are only nonlocal effects, and linearization instead takes place around $I=\delta I$, with $\kappa=(1-Y) / \nu_{\ell}$. The resulting differential equation is therefore $d^{2}\left(\nabla^{2} \delta I\right)-\kappa \delta I=0$, whose solutions are an exponential with different lengthscale, $L=d \sqrt{\frac{\nu_{\ell}}{1-Y}}$. Written together, the physical lengthscale $L$ is described by the following piecewise function:

$$
\frac{L}{d}= \begin{cases}\sqrt{\frac{Y \nu_{\ell}}{Y-1}} & Y>1 \\ \sqrt{\frac{\nu_{\ell}}{1-Y}} & Y<1\end{cases}
$$

Thus, as for the cooperative model, there is again a divergent length scale at $\mu_{\mathrm{s}}$, but in this case there is an asymmetry above/ below $\mu_{\mathrm{s}}$.

\section{Method}

\subsection{Apparatus}

Our apparatus consists of a quasi-2D annular shear cell; ${ }^{20}$ this geometry allows continuous shearing from the inner wall and visual access to the dynamics of all particles. The particles are a bidisperse mixture of circular (60\%) and elliptical (40\%) disks cut from $3 \mathrm{~mm}$ thick PhotoStress Plus PS-3 polymer from the Vishay Measurements Group (bulk modulus $0.21 \mathrm{GPa}$, density $\left.\rho=1.15 \mathrm{~g} \mathrm{~cm}^{-3}\right)$. Using a bidisperse mixture of circular and elliptical disks suppresses crystallization. The circles have diameter $d=5.6 \mathrm{~mm}$ and the ellipses have major and minor axes of $6.8 \mathrm{~mm}$ and $4.7 \mathrm{~mm}$, respectively; we report length measurements scaled by the circle diameter $d$ (also the geometric mean of the two ellipse axes).

A motor (Parker Compumotor BE231FJ-NLCN with a PV90FB 50:1 gearbox) is attached to the inner wall, providing a constant rotational speed. We measure the inner wall shear stress $\tau\left(R_{\mathrm{i}}\right)$ via a torque sensor (Cooper Instruments \& Systems Torque Sensor) attached to the central shaft. As shown in Fig. 1, the stationary outer wall incorporates 52 laser-cut leaf springs. Each of the springs linearly deforms (both radially and tangentially) under stress from the granular material. Via calibrated image processing, ${ }^{21}$ we obtain quantitative measurements of shear $(\tau)$ and normal $(P)$ stresses at each of the 52 spring tips. Values are reported as spatial and temporal averages.

Table 1 summarizes the six datasets spanning four rotation rates and two packing fractions ( $\Phi_{\mathrm{lo}}$ and $\left.\Phi_{\mathrm{hi}}\right)$. All data is collected after the system reaches a steady state. For inner wall rotation speeds $v\left(R_{\mathrm{i}}\right)=0.02,0.2$, and $2 d / \mathrm{s}$, the data are collected after at least one full rotation; for $0.002 \mathrm{~d} / \mathrm{s}$, we wait two hours (1/12 of a rotation) before collecting data. Images for particletracking are collected at $1 \mathrm{fps}(0.02 d-0.2 d$ /frame), except for the slowest ( $0.1 \mathrm{fps}, 0.02 d /$ frame) and fastest ( $4 \mathrm{fps}, 0.5 d /$ frame) runs. For the fastest dataset, we image only one quarter of the whole apparatus, to allow for a faster frame rate. The duration of the experiments is determined by the rotation rate, ranging from 0.5 to $24 \mathrm{~h}$ so that each run completes 1-2 full rotations at steady state. An additional set of images was taken using a 
Table 1 Description of the six datasets. The inner wall rotation $v\left(R_{\mathrm{i}}\right)$ is the speed set by the motor controller. The number of particles is set by hand to one of two values (corresponding to 4 runs at $\Phi_{\text {lo }}$ with 5610 particles and 2 runs at $\Phi_{\text {hi }}$ with 5760 particles). The global packing fraction $\Phi$ is calculated from number of particles, the area of the particles, and the area of the shear cell (including the measured spring wall dilation). The \pm values correspond to errors in the measurement of the particle size and the fluctuations in the spring wall dilation; these are of similar magnitude, and were added in quadrature. Pressure $P$ is calculated from spatially- and temporally-averaging the normal stress measured at the 52 spring wall arms. The \pm values are the standard deviation of these measurements across both space (52 arms) and time. The microscopic timescale $T$ is calculated based on this pressure and known values of $d$ and $\rho$

\begin{tabular}{|c|c|c|c|c|c|c|}
\hline$v\left(R_{\mathrm{i}}\right)[d / \mathrm{s}]$ & 2 & 0.2 & 0.02 & 0.002 & 2 & 0.2 \\
\hline Number of particles & 5610 & 5610 & 5610 & 5610 & 5760 & 5760 \\
\hline$\Phi$ & $0.819 \pm 0.002$ & $0.818 \pm 0.002$ & $0.817 \pm 0.002$ & $0.817 \pm 0.002$ & $0.839 \pm 0.002$ & $0.841 \pm 0.002$ \\
\hline$P[\mathrm{kPa}]$ & $7.5 \pm 0.4$ & $8.0 \pm 0.4$ & $9.2 \pm 0.4$ & $9.3 \pm 0.4$ & $12.0 \pm 1.0$ & $9.2 \pm 0.4$ \\
\hline
\end{tabular}

darkfield polariscope, ${ }^{17}$ allowing us to visualize interparticle forces and their fluctuations. We collected this for an additional dataset with $v\left(R_{\mathrm{i}}\right)=2 d / \mathrm{s}$ and $\Phi_{\mathrm{hi}}$, in order to make measurements both above and below $\mu_{\mathrm{s}}$.

\subsection{Particle tracking}

We track the particles in Matlab using a Hough transform ${ }^{22}$ combined with the Blair-Dufresne particle-tracking code. ${ }^{23}$ Particle speeds are obtained from taking finite differences of the particle locations along these tracks. For the fastest runs, particle tracks immediately adjacent to the inner wall are inaccessible and data is not reported for those positions. To obtain the azimuthal speed profile $v(r)$, we calculate the average tangential speed $v$ within concentric rings of width $0.65 d$ located at different distances from the center. The shear rate profile is calculated using a Fast Fourier Transform (FFT)derivative of the averaged $v(r)$ profile. Fourier-derivatives provide an improvement over finite differences: they are less noisy since each data point is calculated using information from the whole timeseries. The reduced noise is important when taking higherorder derivatives. In polar coordinates, the azimuthal shear rate is given by $\dot{\gamma}(r)=\frac{1}{2}\left(\frac{\partial v}{\partial r}-\frac{v}{r}\right)$.

The resulting speed and shear rate profiles are shown in Fig. 2, for all six datasets. Each datapoint is the average over 100 tracked particles (averaged in space), and approximately 2000 frames (averaged in time). All six speed profiles show strong shear-banding, with the speed falling to nearly zero within about $10 d$ of the inner wall. Note that data close to the inner wall is excluded from plots; this arises where images are too blurred by motion to confidently report quantitative values. We observe that for datasets at the same $\Phi$, the shear rate is greater for higher inner wall rotation rates, when compared at the same location. For the same inner wall rotation rate, higher $\Phi$ have a higher shear rate at same location.

We measure the local packing fraction $\phi$ as a function of $r$ by dividing images into concentric rings of width $2.5 \mathrm{~d}$. Within each of these rings, we calculate the fractional area of the particles detected; approximately 250 particles are used in each average. The results are shown in Fig. 3 for each of the six datasets, all following an approximately exponential decay with the same characteristic length. However, for the same wall rotation rate, runs with higher global $\Phi$ also have a higher local $\phi$ near the inner wall.
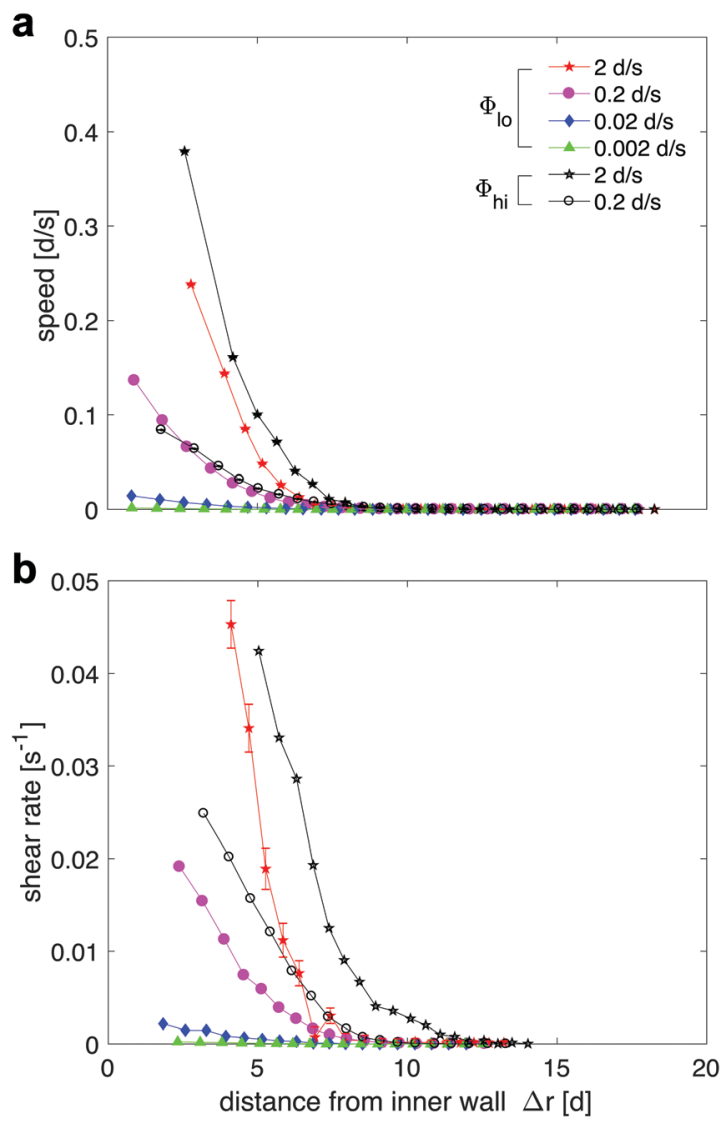

Fig. 2 (a) Speed profiles $v(r)$ and (b) shear rates $\dot{\gamma}$ for all six datasets, measured by particle-tracking. The distance from inner wall is at location $\Delta r=r-R_{\mathrm{i}}$. We estimate the error bars using finite differences, and show typical values for one run. Data files for all figures are available online through DataDryad.

\subsection{Inertial number}

To measure the spatial dependence of the inertial number $I(r)$ (eqn (1)) at each point within the experiment, we combine the measured shear rate $\dot{\gamma}(r)$ for each dataset (Fig. 2b) with the measured (constant) pressure $P$ (Table 1 ). In all cases, the values are azimuthally and temporally averaged. We observe a high value of $I$ near the inner wall, and a low value of $I$ near the outer wall across all six datasets, with values ranging from $10^{-8}$ to $10^{-4}$, falling well within the quasistatic ${ }^{8}$ regime $\left(I<10^{-2}\right)$. These values of $I$ place the majority of our data in the nonlocal regime. 


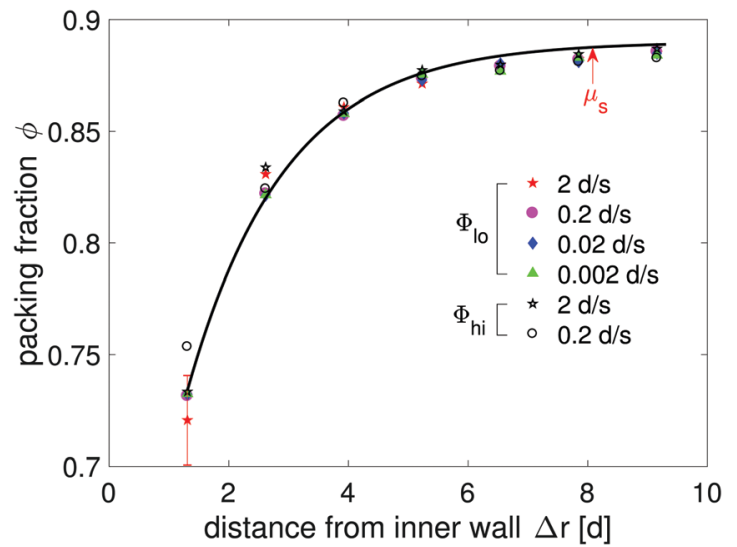

Fig. 3 Local packing fraction profiles $\phi(r)$, for all six datasets. The solid line is an exponential with decay length $r_{0}=(1.67 \pm 0.17) d$, taken from averaging over fits to all six datasets.

\subsection{Shear and normal stress}

By tracking the displacement of the 52 tips on the spring wall boundary (see ref. 21 for details), we measure the shear stress $\tau$ at the inner and outer walls, and the normal stress (pressure) $P$ at the outer wall. We assume that $P$ is constant throughout the system (force balance) and that $\tau(r)$ can be described by accounting for boundary drag in addition to geometric arguments. If no drag with the upper/lower plates were present, then $\tau(r)=S\left(R_{\mathrm{i}} / r\right)^{2}$ where $S$ is the measured shear stress at the inner wall. ${ }^{2}$ We assume that an additional contribution, due to basal friction, is related to the local packing fraction $\phi(r)$. As shown in Fig. 3, $\phi(r)$ takes an exponential form

$$
\phi(r)=\phi_{0}\left[1-\mathrm{e}^{-\Delta r / r_{0}}\right]+\phi\left(R_{\mathrm{i}}\right) .
$$

where $\Delta r \equiv r-R_{\mathrm{i}}$ is the distance from the inner wall. The parameter $r_{0}$ represents the decay length in Fig. 3, and $\phi_{0}=\phi\left(R_{\mathrm{o}}\right)-\phi\left(R_{\mathrm{i}}\right)$ represents the change in packing fraction between the outer wall and the inner wall.

Correspondingly, we write a phenomenological model for the shear stress profile:

$$
\tau(r)=S_{0}\left(\frac{R_{\mathrm{i}}}{r}\right)^{2}+\tau_{0}\left[1-\mathrm{e}^{-\Delta r / r_{0}}\right]
$$

This form is motivated by two main features: the driving from the inner wall $\left(\tau \propto\left(R_{\mathrm{i}} / r\right)^{2}\right)$, plus an exponential decay from basal fraction (eqn (16)). Here, we assume that an additional shear stress term arises from the basal friction between the particles and the base, and is therefore proportional to $\phi(r)$. Since the first term in eqn (17) already accounts for the basal friction of the particles in contact with the inner wall, we only need to add an additional correction to account for the increase in basal friction due to increasing $\phi(r)$. This additional term is therefore proportional to $\left[\phi(r)-\phi\left(R_{\mathrm{i}}\right)\right] \propto\left[1-e^{-\Delta r / r_{0}}\right]$. The value $r_{0}=1.67 d$ comes from Fig. 3 , and we determine parameters $\left(S_{0}, \tau_{0}\right)$ by fitting each of the six datasets to eqn (17) subject to the measured endpoints $\tau\left(R_{\mathrm{i}}\right)$ (from the torque sensor) and $\tau\left(R_{\mathrm{o}}\right)$ (from the leaf springs). The average value $\tau_{0}=250 \pm 30 \mathrm{~Pa}$ is

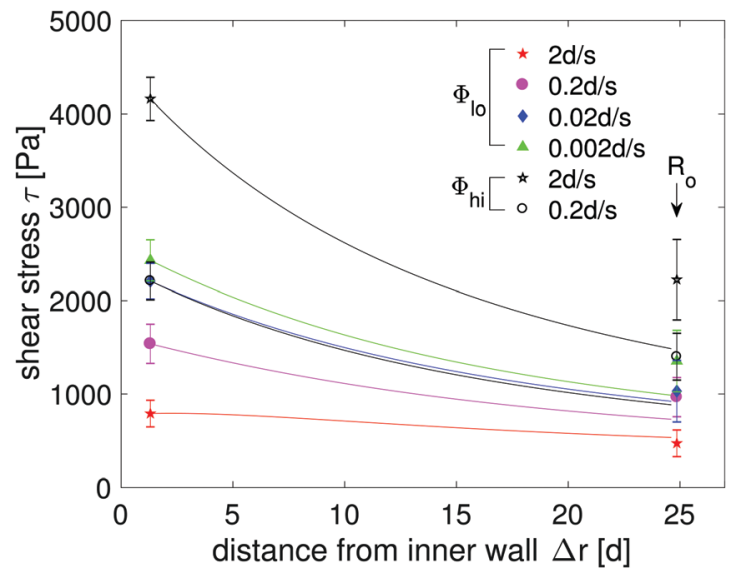

Fig. 4 Modeled shear stress profiles $\tau(r)$, determined from the model given in eqn (17), with $\tau_{0}=(250 \pm 30) \mathrm{Pa}$ and $r_{0}$ taken from Fig. 3. Datapoints at $R_{\mathrm{i}}$ come from the torque sensor, and at $R_{\mathrm{o}}$ from the leaf springs, for each of the six datasets.

then used for all datasets as our model of basal friction, while retaining the six individual values of $S_{0}$ to reflect the driving.

The resulting curves for $\tau(r)$ are shown in Fig. 4. For the same rotation rate, datasets with higher $\Phi$ experience a higher shear stress at the same location. For the same $\Phi$, experiments with lower rotation rates have higher shear stress for the same location.

\section{Results}

\section{$3.1 \mu(I)$ rheology}

The measurements from Sections 2.3 and 2.4 can be plotted parametrically to obtain a graph of $\mu(I)$, as shown in Fig. 5 a. For all six datasets, the inner (faster) part of the flow is located on the right side of the graph, at larger values of $I$. Fig. 5 a provides our first estimate of the value of the yield stress ratio $\mu_{\mathrm{s}}$, which is the upper limit of $\mu(I)$ for the slowest run (rotation rate $0.002 \mathrm{~d} / \mathrm{s}$ ). Because the shear ratio $\mu$ approaches $\mu_{\mathrm{s}}$ for very slow inertial numbers, the upper limit at low- $I$ has previously been taken as a good estimate of $\mu_{\mathrm{s}}{ }^{2}{ }^{2}$ This is approximately $\mu_{\mathrm{s}}=0.26$, a value which will be further confirmed with two additional methods below.

Note the reversal of trends for the two different packing fractions: for $\Phi_{\mathrm{lo}}$, decreasing the rotation rate of the inner wheel raises $\mu$, while the opposite is true at $\Phi_{\mathrm{hi}}$. This is echoed by the measurements of $P$ (Table 1 ) and $\tau$ (Fig. 4). The likely reason for this effect is the dilatancy transition: ${ }^{24}$ denselypacked granular materials dilate under shear, while looselypacked ones compact. As measured by the two-point correlation of free Voronoï volumes in binary disc packings, ${ }^{25}$ the $2 \mathrm{D}$ dilatancy transition occurs between $\Phi_{\mathrm{lo}}$ and $\Phi_{\mathrm{hi}}$, near 0.827 .

\subsection{Fluidity}

Both the cooperative (Section 1.1) and gradient (Section 1.2) models depend on the calculation of the local fluidity. Our experimental measurements of $(\dot{\gamma}, \mu, P)$ in the preceding figures 

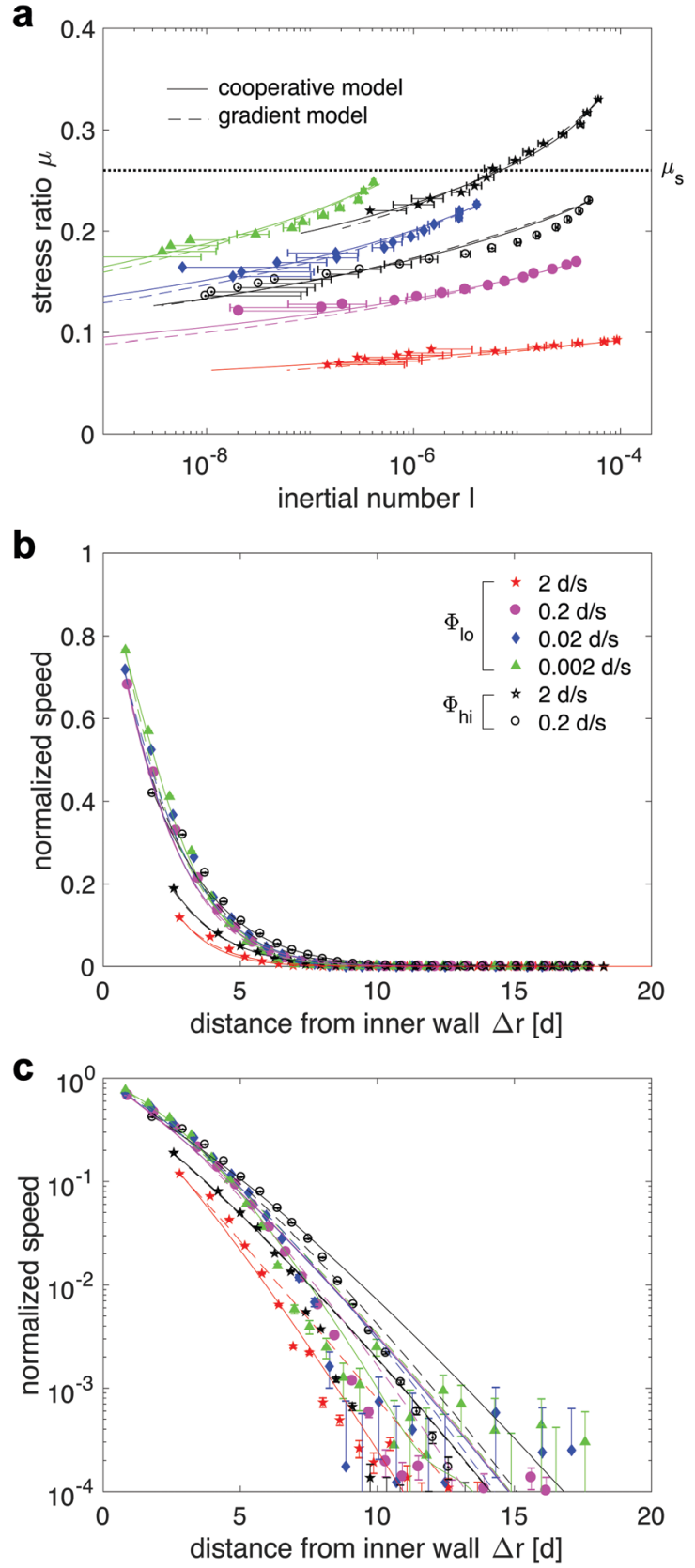

Fig. 5 (a) Stress ratio $\mu$ as a function of inertial number $l$, for all six datasets. The horizontal dotted line indicates the location of $\mu_{\mathrm{s}}=0.26$ to distinguish the local (upper) and nonlocal (lower) regions. Speed profiles $v(r)$ for all six datasets, plotted as (b) raw data on a linear axis and (c) normalized on a logarithmic axis. In all cases, solid lines compare to the cooperative model (Section 1.1) and dashed lines to the gradient model (Section 1.2). For comparison, we calculate relative residuals as $R^{2} \equiv$ $\left\langle\left(a_{\exp }-a_{\mathrm{th}}\right)^{2} /\left(a_{\exp }{ }^{2}\right)\right\rangle$, with the average taken over all data points. For (a): $R^{2}=0.204$ for the cooperative model, and $R^{2}=0.210$ for the gradient model. For (b and c): $R^{2}=0.129$ for the cooperative model, and $R^{2}=0.124$ for the gradient model.

provide values for eqn (3) (cooperative model):

$$
g_{\exp }(r)=\frac{\dot{\gamma}_{\exp }(r)}{\mu_{\exp }(r)} .
$$

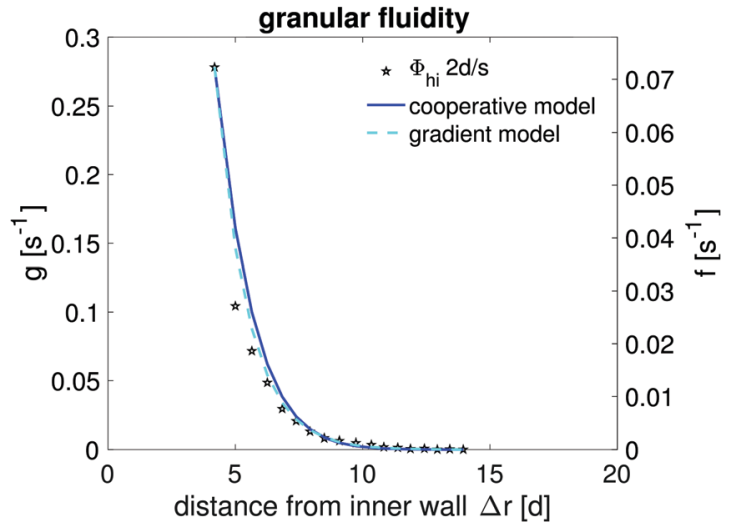

Fig. 6 Experimentally-measured granular fluidity (calculated by eqn (18) and (19)), compared to best fit theoretical curves for both models. The line for the cooperative model $g$ (left axis) is calculated from eqn (6) and the line for the gradient model $f$ (right axis) is calculated from eqn (11). Since the relationship between the two granular fluidity is $g \mu_{\mathrm{s}}=f$ (see eqn (3) and (8)), the left and right axes have been scaled by $\mu_{\mathrm{s}}$ to allow for comparison on a single plot. The dataset was taken at $\Phi_{\mathrm{hi}}$ and $v\left(R_{\mathrm{i}}\right)=2 \mathrm{~d} / \mathrm{s}$.

and eqn (8) (gradient model):

$$
f_{\exp }(r)=\frac{\mu_{\mathrm{s}} \dot{\gamma}_{\exp }(r)}{\mu_{\exp }(r)} .
$$

where only $\mu_{\mathrm{s}}=0.26$ is a fit parameter. The resulting data are shown in Fig. 6: as expected, the system is most fluid near the inner shearing wall. Note that the ratio between the two fluidities $(f, g)$ is just $\mu_{\mathrm{s}}$. Therefore, we can scale the left and right axes so that only a single set of data points is shown for both.

\subsection{Comparison to models}

For the cooperative model, we obtain $g(r)$ by solving eqn (6) using the Matlab ordinary differential equation (ODE) - solver $b v p 4 c$. The two boundary conditions are taken as the value of $g_{\text {exp }}(r)$ nearest the inner wall, and $g\left(R_{\mathrm{o}}\right)=0$. Using LevenbergMarquardt optimization, we obtain the fitting parameters $A$ and $b$ from the dataset with $v\left(R_{\mathrm{i}}\right)=2 d / \mathrm{s}$ and $\Phi_{\mathrm{hi}}$. These two parameters $(A, b)$ are used to plot all of the solid lines in Fig. 5 and 6. See Table 2 for the values and uncertainties.

For the gradient model, the $\dot{\gamma}$ in eqn (11) is written as a function of $f$, and we solve for $f(r)$ (again using $b v p 4 c$ ). The boundary conditions of $f(r)$ are determined in the same way as for the cooperative model. Using the dataset with $v\left(R_{\mathrm{i}}\right)=2 d / \mathrm{s}$ and $\Phi_{\mathrm{hi}}$, we simultaneously fit the parameters $a$ and $\ell$ using Levenberg-Marquardt optimization. This set of parameters is used to plot all of the dashed lines in Fig. 5 and 6.

Using the parameters in Table 2, we solve the cooperative model (eqn (6), using $\mu_{\mathrm{s}}, A, b$ ) and the gradient model (eqn (11), using $\mu_{\mathrm{s}}, \ell, a$ ) for all six runs, to get the granular fluidity $(g(r)$ and $f(r)$, respectively).

Recall that the values of $\mu(r)$ were calculated in Section 3.1. For the cooperative model, $I(r)=g(r) \mu(r) T$ and for the gradient model $g(r)$ is replaced by $f(r) / \mu_{\mathrm{s}}$. The resulting parametric plots of $\mu(r) v s . I(r)$ are shown in Fig. 5a, with the cooperative model represented by solid lines, and the gradient model by dashed 
Table 2 Fitting parameters for both models, with \pm values representing the sensitivity range. The sensitivity for $\mu_{\mathrm{s}}$ (identical for both models) is taken from the full-width-half-maximum of the peak in the inset to Fig. 7. For the four parameters $(A, b, \ell, a)$, the sensitivity is determined from the run with $\Phi_{\mathrm{hi}}$ and $2 d / \mathrm{s}$ by holding one parameter fixed and allowing the residual to vary up to $R^{2}=0.3$ (rather than the best-fit $R^{2}=0.2$ shown in Fig. 5a). Similarly, for the lengthscale parameter $\nu_{\ell}$, the sensitivity range is for an increase from $R^{2}=0.02$ to 0.03 for the data and fit given in Fig. 7

\begin{tabular}{|c|c|c|c|}
\hline \multirow[b]{2}{*}{$\mu_{\mathrm{s}}$} & \multicolumn{3}{|c|}{ Cooperative model } \\
\hline & $A$ & & $b$ \\
\hline \multirow{2}{*}{$0.26 \pm 0.01$} & 0.402 & & $1.1 \pm 0.5$ \\
\hline & \multicolumn{3}{|c|}{ Gradient model } \\
\hline$\mu_{\mathrm{s}}$ & $\ell$ & $a$ & $\nu_{\ell}$ \\
\hline $0.26 \pm 0.01$ & $1.02 \pm 0.02$ & $7.2 \pm 4.3$ & $0.46 \pm 0.03$ \\
\hline
\end{tabular}

lines. Both models show good agreement with the data, with similar $R^{2}$ residuals.

We can also compare the speed profiles, as has been done for two other geometries in ref. 15. The predicted speed profile $v(r)$ is obtained by numerically integrating $\dot{\gamma}=\mu(r) g(r)$ from the outer wall $R_{\mathrm{o}}$ to the location $r$ (correspondingly, $g(r)$ is replaced by $f(r) / \mu_{\mathrm{s}}$ for the gradient model). The resulting curves are compared with the data on both logarithmic and linear axes in Fig. $5 \mathrm{~b}$ and c. Again, we observe that both models agree well with the data, with no significant difference in the residuals for the two models.

Notably, the comparisons in Fig. 5 were made using a single set of parameters, making them inherent properties of the granular material. As can be seen from the sensitivity ranges given in Table 2, both nonlocal parameters $(A, \ell)$ are much better-constrained by the data than the local parameters $(b, a)$. This is because we have collected the majority of our data in the nonlocal region.

\subsection{Nonlocal lengthscale}

To directly test the prediction of a diverging lengthscale ( $\xi$ or $L$ ) at $\mu_{\mathrm{s}}$ (eqn (7) and (15)), we calculate these quantities from the dataset that spans both the local and nonlocal regimes $\left(\Phi_{\mathrm{hi}}\right.$ and rotation rate $2 d / \mathrm{s}$ ). The lengthscale $\xi$ is determined from eqn (6) using the experimentally-determined values of $g_{\exp }$ and its Laplacian, with $g_{\text {loc }}$ taken from eqn (5) using $\mu(r)$ and the fitting parameter $b$ from Table 2 .

The Laplacian term in eqn (6) requires taking a second derivative of the fluidity (either $g$ or $f$ ), which is itself calculated from $\dot{\gamma}(r)=\frac{1}{2}\left(\left|\frac{\partial v}{\partial r}\right|+\frac{v}{r}\right)$. This requires taking three derivatives of our discretely-sampled $v(r)$ data (see Fig. 2). Because this is numerically-challenging, we use two complementary methods: the analytical method takes analytical derivatives of an empirical fit to $v(r)$ and the numerical method uses FFT-differentiation. The empirical fit to $v(r)$ is shown in Fig. 7a, given by:

$$
v(r)=v_{0} \exp \left[\alpha_{3} r^{3}+\alpha_{2} r^{2}+\alpha_{1} r+\alpha_{0}\right] .
$$

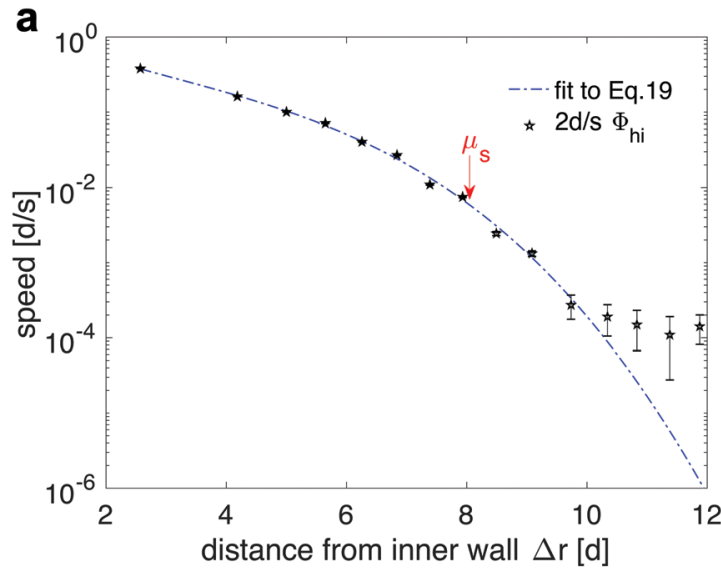

b

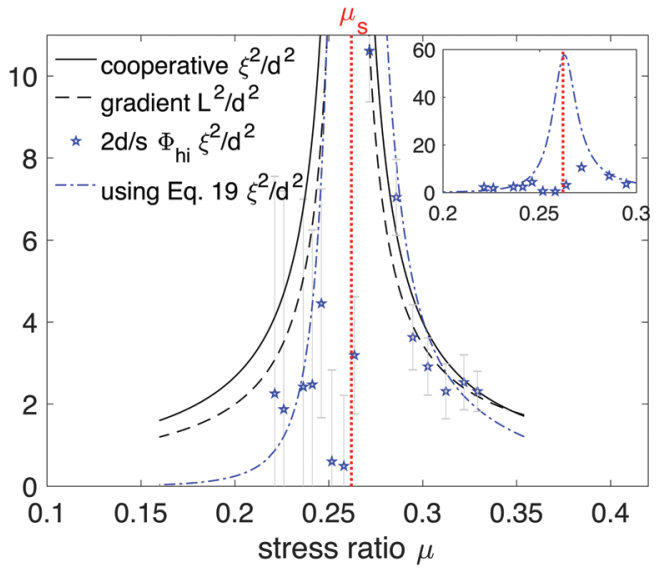

Fig. 7 (a) Speed profile $v(r)$ for the dataset with $\Phi_{\text {hi }}$ and rotation rate $2 d / s$. The dash-dotted line is given by a fit to eqn (20) with $\alpha_{3}=-0.0118, \alpha_{2}=$ $-0.1096, \alpha_{1}=-0.8374$, and $\alpha_{0}=0.6512$, using the filled data points (above $\mu_{\mathrm{s}}$ ). (b) Comparison of the measured lengthscale (blue) to the two models (black), scaled by particle diameter $d$. Both of the models have a divergence near the value $\mu_{\mathrm{s}}=0.26$, as expected from Fig. $5 \mathrm{a}$. For $\mu>\mu_{\mathrm{s}}$ (local regime), the relative residuals are $R^{2}=0.053$ for the cooperative model (black solid line) and $R^{2}=$ 0.092 for the gradient model (black dashed line), based on a comparison with the analytic derivative (blue dash-dotted line). For $\mu<\mu_{\mathrm{s}}$ (nonlocal regime), these same residuals are quite large: $R^{2}=1.617$ for the gradient model, $R^{2}=$ 3.819 for the cooperative model.

In applying the analytical method, we do not use the experimental fluidity data $g_{\exp }$ directly, but instead use this functional form for the calculations.

The lengthscale $L$ for the gradient model is calculated similarly, using eqn (14). Note that eqn (6) and (14) both express the lengthscale ( $\xi$ or $L$, respectively) in terms of the ratio of a nonlocal part $\left(g-g_{\text {loc }}\right.$ or $\left.\delta I \equiv I-I_{\mathrm{loc}}\right)$ to the Laplacian of the total $(g$ or $I)$. This illustrates the association of $I$, itself, as a fluidity-like quantity. Because of the definition of $Y$ (see eqn (8) and vicinity), these two lengthscales are identical. In examining the shape of $L(\mu)$, there is still one free parameter to fit. We obtain $\nu_{\ell}=0.46$ by fitting eqn (15) to the numerical experimental data (blue stars in Fig. 7b) for $\mu>\mu_{\mathrm{s}}$.

The results of these lengthscale calculations give $\xi(r)$, as either a curve (the analytical method, blue dash-dotted line) or a set of discrete points (the numerical method, blue stars), which can be plotted parametrically against $\mu(r)$ to provide $\xi(\mu)$. 
These two datasets are shown in blue in Fig. 7b. While the numerical method works well only for $\mu>\mu_{\mathrm{s}}$ (the local regime), it suggests a growing lengthscale near the same $\mu_{\mathrm{s}}=0.26$ observed in Fig. 5a. The analysis using the analyticallyderived measurement confirms the presence of a clear peak in $\xi(\mu)$ near $\mu_{\mathrm{s}}=0.26$, spanning both the local and nonlocal regimes. These results provide a second, kinematic, verification of the value of the yield stress ratio $\mu_{\mathrm{s}}$.

Finally, we compare these measured lengthscales to the predictions of the two models, using the parameter values given in Table 2. For the cooperative model, the comparison is provided directly by eqn (7), while for the gradient model, the prediction requires solving eqn (15). We find that the predictions from the two models are of similar quality above $\mu_{\mathrm{s}}$ (local regime), as shown in Fig. 7b. Below $\mu_{\mathrm{s}}$ (nonlocal regime), the gradient model (dashed line) provides a better fit to the observed length scales (blue dash-dotted line), through the inclusion of an additional fit parameter $\nu_{\ell}$, which directly controls the shape of $L(\mu)$, which in turn affects $\xi(\mu)$.

\subsection{Yield stress ratio $\mu_{\mathrm{s}}$}

A third, completely independent, method of determining the yield stress ratio $\mu_{\mathrm{s}}$ arises from an examination of the force chain fluctuations. ${ }^{20}$ We use the variance of the force chain intensity as a semi-quantitative proxy measurement for the heterogeneity of the force transmission. As shown in the inset in Fig. 8, the force chains are spatially heterogeneous (and also temporally heterogeneous, not shown). To quantify the radial profile of these fluctuations, we divide a series of images into concentric rings. Within each of these rings, we measure the variance of all measured light intensity values (across both space and time).

In Fig. 8, we parametrically plot the measured variance against the value of $\mu$ measured within that ring. We observe

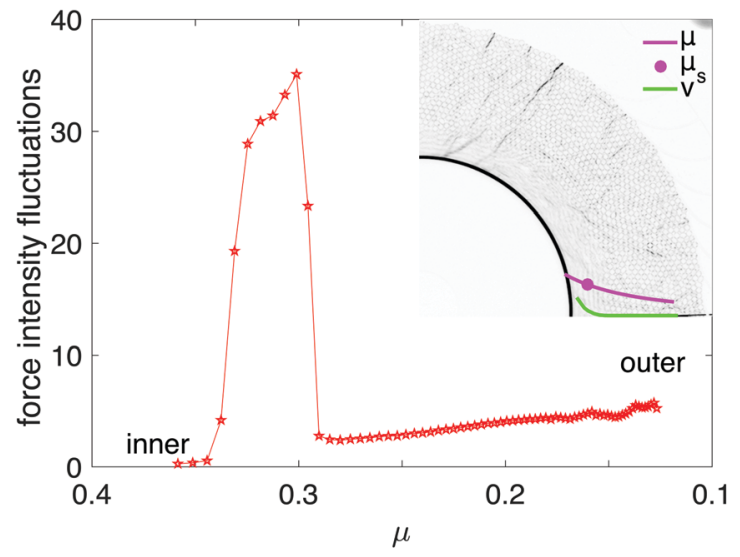

Fig. 8 The variance of the light (force) intensity within concentric rings, plotted parametrically against the value of $\mu$ measured within that ring. The sharp drop is at $\mu_{\mathrm{s}}=0.29$. Inset: Image of force chains, inverted for clarity, so that dark particles are those experiencing more force. Data collected at $\Phi_{\text {hi }}$ and at rotation rate $2 d / s$. Upper line (magenta) overlays the measured $\mu(r)$ with $\mu_{\mathrm{s}}=0.29$ marked by the circle. Lower line (green) overlays the measured $v(r)$ for comparison. that near the inner wall (large $\mu$, left side of plot), the fluctuations grow even as the velocity (also fluidity) drops. This is the local regime, where the cooperative length is increasingly long. At $\mu_{\mathrm{s}}=0.29$, however, the fluctuations abruptly fall as the granular material enters the nonlocal regime. A similar effect was observed by, ${ }^{26}$ in which simulations showed that temporal fluctuations of pressure were largest near spatial locations which were also close to the jamming point. This provides a new physical interpretation of the nature of the change in material properties in the vicinity of $\mu_{\mathrm{s}}$ : a susceptibility to force chain fluctuations.

Interestingly, this value of $\mu_{\mathrm{s}}$ is quite close, but does not precisely agree with the value $\left(\mu_{\mathrm{s}}=0.26\right)$ obtained from particle kinematics and stress measurements. This small difference likely arises from the change in boundary conditions for the two types of experiments: the particle-tracking experiments are performed with a different upper boundary (the upper layer above the particles is clear plastic) from the photoelastic experiments (polaroid sheet). This change in materials likely adjusts the value of $\tau_{0}$ in eqn (17) by a small amount, accounting for the change in drag. We have chosen to use $\mu_{\mathrm{s}}=0.26$ for the model comparison done in Section 3.3, since this corresponds to the boundary conditions used for the $v(r)$ measurements. Had we chosen to use $\mu_{\mathrm{s}}=0.29$, this would disagree with the peak observed in the inset to Fig. $7 \mathrm{~b}$, but it would not significantly affect the $\mu(I)$ results.

\section{Conclusions}

In this paper, we presented new experiments on granular rheology which validate the efficacy of two nonlocal models in describing flow properties. A key advance was the introduction of leaf spring boundaries to provide stress measurements rather than simply relying on particle kinematics. This method will be particularly helpful in future experiments without photoelastic particles (which could otherwise measure the stress), where it is desirable to monitor the spatial and temporal fluctuations of the boundary stresses. By making measurements in a photoelastic system, we additionally uncovered a new interpretation for the model's assumption of a diverging length scale at the yield stress ratio $\mu_{\mathrm{s}}$. We observe that this corresponds to a drop in the susceptibility to force chain fluctuations as the material goes from the local to nonlocal regimes.

In order to make direct, quantitative comparisons between experiments and two popular nonlocal models, the availability of boundary stress measurements allowed us to incorporate a drag term to account for basal friction. To test the applicability of the two models, we used one of six runs to determine the fit parameters; these parameters were then capable of fitting the five other datasets across four different rotation rates (spanning four orders of magnitude in inertial number $I$ ) and two packing fractions. Furthermore, we directly tested the presence of a growing length scale in the vicinity of $\mu_{\mathrm{s}}$, and found it to be consistent with both models. This observation provides additional support for why both models have been successful under 
a variety of circumstances. ${ }^{2-4,16,27}$ Additionally, we find that the model is able to capture the dilatancy transition, without explicitly including it.

Importantly, we find that while both models can quantitatively describe the experimental data, the cooperative model has one fit parameter fewer. This favors its choice where only $\dot{\gamma}(r)$ and $\mu(I)$ are concerned, but the additional parameter $\left(\nu_{\ell}\right)$ in the gradient model allows it to better-fit the nonlocal lengthscale for $\mu<\mu_{\mathrm{s}}$ (the nonlocal regime). Further tests in spatially heterogeneous and/or unsteady situations would be important to better-distinguish the two models.

\section{Conflicts of interest}

There are no conflicts to declare.

\section{Acknowledgements}

We thank Dave Henann, Ken Kamrin, Philippe Claudin and Dave Schaeffer for useful discussions about the project, and Austin Reid for inspiring the boundary wall designs. We are grateful to the National Science Foundation (NSF DMR-1206808 and DMS-1517291) and the International Fine Particle Research Institute (IFPRI) for financial support.

\section{References}

1 Y. Forterre and O. Pouliquen, Annu. Rev. Fluid Mech., 2008, 40, 1-24.

2 K. Kamrin and G. Koval, Phys. Rev. Lett., 2012, 108, 178301.

3 M. Bouzid, M. Trulsson, P. Claudin, E. Clément and B. Andreotti, Phys. Rev. Lett., 2013, 111, 238301.

4 M. Bouzid, A. Izzet, M. Trulsson, E. Clément, P. Claudin and B. Andreotti, Eur. Phys. J. E: Soft Matter Biol. Phys., 2015, 38, 125.

5 T. Barker, D. Schaeffer, M. Shearer and J. Gray, Proc. R. Soc. A, 2017, 473, 20160846.

6 Q. Zhang and K. Kamrin, Phys. Rev. Lett., 2017, 118, 058001.
7 C. S. Campbell, Annu. Rev. Fluid Mech., 1990, 22, 57-90.

8 I. Goldhirsch, Annu. Rev. Fluid Mech., 2003, 35, 267-293.

9 C. S. Campbell, Powder Technol., 2006, 162, 208-229.

10 G. Koval, J.-N. Roux, A. Corfdir and F. Chevoir, Phys. Rev. E: Stat., Nonlinear, Soft Matter Phys., 2009, 79, 021306.

11 G. MiDi, Eur. Phys. J. E: Soft Matter Biol. Phys., 2004, 14(4), 1.

12 X. Cheng, J. B. Lechman, A. Fernandez-barbero, G. S. Grest, H. M. Jaeger, G. S. Karczmar, M. E. Mobius and S. R. Nagel, Phys. Rev. Lett., 2006, 96, 38001.

13 K. Nichol, A. Zanin, R. Bastien, E. Wandersman and M. van Hecke, Phys. Rev. Lett., 2010, 104, 078302.

14 K. Reddy, Y. Forterre and O. Pouliquen, Phys. Rev. Lett., 2011, 106, 108301.

15 D. L. Henann and K. Kamrin, Proc. Natl. Acad. Sci. U. S. A., 2013, 110, 6730-6735.

16 K. Kamrin and D. L. Henann, Soft Matter, 2015, 11, 179-185.

17 K. E. Daniels, J. E. Kollmer and J. G. Puckett, Rev. Sci. Instrum., 2017, 88, 051808.

18 F. Da Cruz, S. Emam, M. Prochnow, J.-N. Roux and F. Chevoir, Phys. Rev. E: Stat., Nonlinear, Soft Matter Phys., 2005, 72, 021309.

19 K. Kamrin and G. Koval, Comput. Part. Mech., 2014, 1, 169-176.

20 D. Howell, R. Behringer and C. Veje, Phys. Rev. Lett., 1999, 82, 5241.

21 Z. Tang, T. A. Brzinski and K. E. Daniels, Powders Grains, 2017, 140, 03035.

22 Hough Transform, https:/www.mathworks.com/help/images/ ref/imfindcircles.html/.

23 D. Blair and E. Dufresne, The Matlab Particle Tracking Code Repository, http://site.physics.georgetown.edu/matlab/.

24 B. P. Tighe, Granular Matter, 2014, 16, 203-208.

25 S.-C. Zhao, S. Sidle, H. L. Swinney and M. Schröter, EPL, 2012, 97, 34004.

26 S. Chialvo, J. Sun and S. Sundaresan, Phys. Rev. E: Stat., Nonlinear, Soft Matter Phys., 2012, 85, 021305.

27 M. van Hecke, C. R. Phys., 2015, 16, 37-44. 